\section{P-169 VIRTUALLY UNKNOWN VIRTUAL LEARNING. TAKING LEARNING PLATFORMS INTO HOSPICE EDUCATION}

Jenny White. Garden House Hospice Care, Letchworth, Hertfordshire

10.1136/spcare-2021-Hospice. 185

Background An annualised training programme was delivered for all acute, community and care home staff in classrooms through short sessions, half days, full days and extended courses. Internal mandatory training only was delivered by elearning and supplemented for clinical staff through a face-toface study day. With the upsurge of a global pandemic, faceto-face training was halted and electronic learning was introduced to deliver much needed education safely and quickly to acute, care home and community staff. There were concerns about the 'safety' of delegates when delivering emotional content remotely.

Aims

- To deliver flexible, high quality short training sessions to meet the rapidly changing needs of staff.

- To deliver end-of-life care training alongside COVID-19 specific requirements.

- To record mandatory training topics for staff and volunteers to reduce 'footfall' within the hospice.

\section{Progress}

- Established a dedicated 'virtual' training room and supported staff to utilise the technology.

- Needs identified with CCG and care homes.

- Training delivered as required including for night staff and at weekends.

- Developed virtual delivery of Foundation, Intermediate and Advanced Communication Skills attracting staff from across the country.

- Delivered an education programme alongside council funded trainers, as well as in conjunction with the ICS for frontline staff as part of the local hospice education consortium.

- Facilitated education for the regional ambulance trust.

- Delivering training for a national charity on end-of-life care.

- Recorded MCA and safeguarding videos to update clinical staff working remotely.

Results Figures showed an increase of external attendees at training (184\%). Virtual training offers a flexible and widereaching approach. Concerns remain over the sensitive nature of training and attendees being effectively supported. However, no anecdotal evidence or evaluations showed staff felt unsupported.

Conclusion Virtual education has a significant role in delivering end-of-life care education allowing greater numbers of staff to access flexible training from a wider geographic area.

\section{P-170 ENGAGING THE PALLIATIVE CARE CLINICAL WORKFORCE THROUGH ONLINE LEARNING. A SUCCESS}

Jennifer Spears, Maggie Fitzgerald, Jo Brady. North London Hospice, London, UK

\subsection{6/spcare-2021-Hospice.186}

Background Achieving engagement of clinicians with regular voluntary teaching sessions is challenging. Voluntary attendance of teaching sessions at the hospice has historically been low and uncoordinated between teams, with momentum difficult to establish; timings not always suiting a workforce with different commitments, shift patterns and geographical workplaces. Yet continuous professional development is a requirement for all (General Medical Council. Continued professional development: guidance for all doctors, 2012; Nursing and Midwifery Council. The code: Professional standard of practice and behaviour for nurses, midwives and nursing associates, 2018).

Aims Two nurses and a doctor were tasked with reintroducing clinical teaching for the hospice's community CNS teams. They decided delivery would be online using Zoom (due to COVID-19), recognising the opportunity this gave to expand access beyond the community clinical nurse specialist teams to all the different hospice clinical teams and sites.

Methods Responsibility was shared for arranging speakers and for gaining support for the new programme from team leads. The learning and development lecturer/CNS took responsibility for managing Zoom, creating QR evaluation codes, storing recordings on the shared hospice server, keeping attendance lists and providing feedback for speakers.

Results November - June 2021:

- Fifteen 45-minute sessions covering a wide range of topics; delivered fortnightly, commencing November 2020.

- 303 recorded attendances: 203 nurses, 20 doctors, 26 allied health professionals, 13 healthcare assistants, 22 trainee nursing associates, 7 Social Work, 19 paramedics, 3 Other. Attendance was across all hospice sites and clinical teams, including six guest paramedics. Some teams had higher percentages of team attendance than others.

- 270 attended live and 43 watched recordings.

- Recorded sessions were also shared with the local hospital palliative care team.

- 34\% completed QR evaluations. Positive feedback, with demonstration of how new knowledge can be transferred to practice.

Conclusion Teaching and learning online has facilitated increased engagement in overall numbers across all the hospice clinical teams and sites, with easier access to internal and external speakers. Moving forward, continued encouragement from management to embed regular attendance across teams may be beneficial, to encourage those who have not yet made attendance habitual.

\section{P-171 NURSE PRESCRIBING: A REAL COMMUNITY OF PRACTICE IN THE VIRTUAL WORLD}

Nigel Dodds. St Christopher's Hospice, London, UK

10.1136/spcare-2021-Hospice. 187

Continuing professional development for non-medical prescribers is recognised as being pivotal in maintaining up-to-date knowledge and skills influencing prescribing competence (Weglicki, et al., 2015). In palliative and end-of-life care, pharmacology and prescribing are rapidly changing and require regular CPD in order to keep up-to-date with the latest developments. Non-medical prescribing is a comparatively new innovation to nursing practice, and within the hospice setting. Therefore, nurse prescribers need mentorship from 
experienced prescribers, as well as the encouragement from nurse leaders to be confident prescribers themselves and enhance patient care (Wilson, et al.,2014).

At St Christopher's, we have encouraged our specialist nurses to undertake academic training to become independent nurse prescribers, and have seen practice flourish. A recent audit of anticipatory prescribing demonstrated the value of nurse prescribers in hospice care, in that $55 \%$ of all anticipatory prescribing was undertaken by nurse independent prescribers. This highlights the pressing need for continuing professional development.

Until 2020, St Christopher's supported our own nurses to attend a national study day to provide pharmacology and prescribing updates, but with the assault of COVID-19, we needed to think differently about how to deliver this. In doing so, we developed a monthly online community of practice which has grown in popularity. Joining this we have over 100 nurses working in palliative and end-of -life care who are non-medical prescribers. Once a month, using video conferencing technology, members of the community join us from all the four nations of the UK, to learn together, share knowledge and access the expertise of invited speakers.

This presentation will outline the significant role of nurse prescribers within the hospice setting, and highlight the need to ensure the availability of CPD. In doing so, we will highlight one such approach to providing $\mathrm{CPD}$, along with raising the national profile of this important work.

\section{P-172 MAINTAINING THE CONFIDENCE OF NON-MEDICAL PRESCRIBERS - BENEFITS OF A VIRTUAL NMP FORUM}

${ }^{1}$ Jo Poultney, ${ }^{2}$ Karen Badgery. ${ }^{1}$ South Warwickshire Foundation Trust, Warwick, UK; ${ }^{2}$ Myton Hospice, Coventry, UK

\subsection{6/spcare-2021-Hospice.188}

Background An increasing number of specialist palliative care nurses are embarking on training to become a prescriber. Non-medical prescribers (NMPs) play a very important role in supporting patients and allowing a more efficient and responsive service (Cope, Abuzour, Tully, 2016). Despite the training being rigorous, one in 10 prescribers say they lack confidence in their new skill (Nurse prescribers competent but not confident. Nursing Standard. 20, 30, 10-10. doi: 10.7748/ns.20.30.10.s15). If appropriate support is not provided to this group of professionals, benefit of their training may be lost. In 2016 a monthly NMP forum was set up in Coventry and Warwickshire to provide a supportive environment to share experiences. Ten nurses attended on average each month, supported by a consultant and pharmacist.

At the beginning of the pandemic it was decided to offer the opportunity to meet via Teams. 88 NMPs have now requested to join the invite list. On average between 25 and 30 attend each meeting. Being on Teams has enabled far more people to be able to join from across the county. A survey was carried out to understand more about the group and to ensure that it was meeting the needs of those who attend.

Results People who attend vary between being a prescriber for $>10$ years to those just embarking on their training. Having members of other specialist palliative care teams in different areas allows those who attend to see similarities/differences in working practice and take best practice back to their area. They report valuing the opportunity to discuss complex symptoms and to share different ways to manage them. It is seen as a 'safe place' where they can receive support, knowledge, education and supervision to support their role as an NMP. $85 \%$ report that the meeting meets their learning needs. $70.3 \%$ more likely to attend if meeting continues to be held virtually

Conclusion The results from the survey have reinforced the plan to continue offering these sessions in their current format.

\section{P-173 AN ICS WIDE VIRTUAL NON-MEDICAL PRESCRIBER FORUM: A PILOT PROPOSAL}

Abi Ponnampalam. Farleigh Hospice, Chelmsford, UK

\subsection{6/spcare-2021-Hospice. 189}

Background Non-medical prescribers (NMPs) have a significant role to play in providing effective and timely access to medications for patients at the end-of-life (Ziegler, Bennett, Mulvey et al., 2018; Weeks, George, Maclure, et al., 2016). There is increasing recognition of the positive contribution of NMPs for patient care especially during out-of-hours periods (Webb \& Gibson, 2011).

Worryingly, not all NMPs feel prepared to prescribe after qualifying with some choosing not to at all (Ziegler, Bennett, Blenkinsopp, et al., 2015; Ryan-Woolley, McHugh, Luker, 2007). Prescribing decision making can be perceived as complex with recent research supporting the role of peer mentoring and support in developing professional practice (Latham \& Nyatanga, 2018; Bowskill, Meade, Lymn, 2014).

Peer support has been shown to improve NMP confidence as well as positively influence prescribing (Otway, 2013; McIntosh, Stewart, Forbes-McKay et al., 2016). As palliative care services adapt to the challenges of the pandemic, virtual forums offer a platform for peer support and cross-organisational learning without the limitations of in-person meetings (Dunleavy, Preston, Bajwah, et al., 2021).

We propose a virtual NMP forum based on Lave and Wenger's 'Community of Practice', a social learning theory where knowledge develops collectively through collaboration within a group they enhance their skills and confidence in their roles as NMPs. By opening up the forum to specialist palliative care NMPs working across organisations and care settings, we hope to foster closer professional relationships and support innovation with the aim of developing services to meet the needs of the local community.

Aim To pilot and evaluate a virtual NMP Forum based on a 'Community of Practice' model across a newly designated Integrated Care System spanning three independent hospices, community and acute trust providers of specialist palliative care services.

Methods Bi-monthly virtual meetings will be held to provide opportunities for peer support, sharing best practice and networking across organisations and care settings. The session design will be fluid with opportunity to modify based on NMP feedback. Sessions will be evaluated for relevance to their role as NMPs, ease of attendance and peer support.

We hope to successfully pilot and incorporate this model within the region. 